\title{
LBBB: The ECG Patterns and Cardiac Function in Patients With and Without Coronary Artery Disease
}

\author{
Marwan Badri, William Kornberg, James F. Burke, \\ Peter R. Kowey and Li Zhang \\ Lankenau Medical Center, \\ Lankenau Institute for Medical Research \\ USA
}

\section{Introduction}

Left bundle branch block (LBBB) is a common conduction abnormality. Its prevalence increases from $0.4 \%$ at age 50 to $6.5 \%$ at age 80 . (Eriksson, Hansson et al. 1998) LBBB is seen in many diseases such as coronary artery disease (CAD), hypertension, valvular heart disease, non-ischemic cardiomyopathies and conduction tissue defects/degenerations. (Nisse 1929; Jain and Mehta 2003) Identifying CAD in patients with LBBB has been the subject of many studies and remains a clinical challenge.

The conduction abnormality caused by LBBB alters the ventricular activation sequence, resulting in distortion of the QRS complex, and secondary changes in the ST segment and Twave on the surface ECG. Presence of LBBB is known to mask the ECG features of CAD. Since approximately $50 \%$ of LBBB patients have underlying CAD, identifying the ECG differences between $C A D$ and non-CAD patients with $\mathrm{LBBB}$ would have considerable clinical impact in risk stratifying LBBB and in the detection of underlying CAD.

Hayat et al reported that myocardial blood velocity was markedly reduced in LBBB patients with CAD compared with those without. (Hayat, Dwivedi et al. 2008) Whether CAD can modify the QRS-ST-T patterns over time in the presence of LBBB is unclear. The first part of this study, therefore, was designed to compare QRS morphology and ST-T changes between CAD and non-CAD patients to determine if there were any subtle ECG pattern differences between the two groups.

The consequence of LBBB is the delay of left ventricular (LV) contraction, altered septal motion, and reduced diastolic filling time.(Strauss, Selvester et al.; Grines, Bashore et al. 1989) These effects impair the pump function and stimulate negative LV remodeling. (Sweeney, van Bommel et al.) An inverse relationship between the QRS duration and left ventricular ejection fraction has been observed in LBBB patients in the presence of mechanical asynchrony. (Roshan, Pati et al. 2008) This finding provides the basis for cardiac resynchronization therapy (CRT) in heart failure patients. (Strauss, Selvester et al.) It is unclear, however, if such a relationship is more pronounced when underlying CAD exists. 
The second part of this study, therefore, was to examine the correlation between QRSd and LV ejection fraction (EF) in patients with $\mathrm{LBBB}$, and to determine if this relationship is altered by CAD.

\section{Methods}

\subsection{Materials and methods}

A retrospective study in accordance with compliance guidelines was conducted after obtaining the approval of the Institutional Review Board of the Lankenau Medical Center. Patients' identifiable information was removed for the data analysis.

The LBBB diagnosis was first made by the automated interpretation of the ECG system. Then it was over-read by a cardiologist to confirm the diagnosis. LBBB ECGs were retrieved from the ECG library and from patients who underwent cardiac catheterization between January $1^{\text {st }} 2005$ and June 30 $0^{\text {th }}$ 2010. Electronic medical records were reviewed to extract demographic and clinical information. A cardiac catheterization database containing patients who had undergone diagnostic coronary angiography, percutaneous coronary intervention (PCI) and/or coronary artery bypass grafting (CABG) was interrogated. Patients were classified as having CAD if they had history of PCI, CABG or if they had $\geq 50 \%$ luminal diameter stenosis of a major coronary artery on angiography.

\subsection{ECG evaluation}

To determine whether LBBB ECG morphology is different between CAD and non-CAD groups, the resting supine 12-lead ECG (AC filter $60 \mathrm{~Hz}, 25 \mathrm{~mm} / \mathrm{sec}, 10 \mathrm{~mm} / \mathrm{mV}$ ) was evaluated for six aspects: 1) Pathologic $Q$ waves. 2) QRS duration (QRSd), amplitude and axis. 3) Among patients with leftward QRS axis $\geq-45$ degrees, QRS morphology was further evaluated for the presence of left anterior fascicular block (LAFB) pattern (Figure 1)

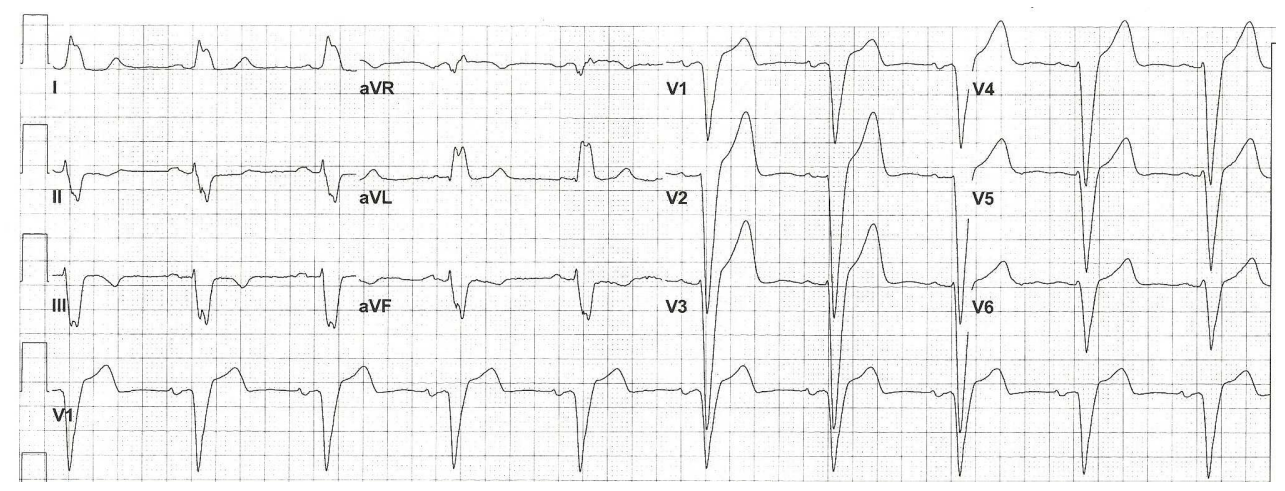

ECG from a 69-year-old man with CAD and EF 31\% demonstrating QRS-ST-T pattern typical to divisional LBBB: QRSd $=188 \mathrm{~ms}$. The LAFB pattern is shown as QRS axis $>-45$ degree, $\mathrm{rS}$ in II, III and aVF with deflection of $\mathrm{S}_{\mathrm{III}}>\mathrm{S}_{\mathrm{II}}$; and broad and notched $\mathrm{R}$ wave in I and $\mathrm{qR}$ in aVL with $\mathrm{R}_{\mathrm{aVL}}>\mathrm{R}_{\mathrm{I}}$; Poor $\mathrm{R}$ progression with $\mathrm{rS}$ in $\mathrm{V} 6$ and $\mathrm{T}$ wave opposite to the direction of QRS complex in precordial leads.

Fig. 1. Poor R progression V1-6 in a CAD patient with LBBB 
4) Low QRS voltage in the limb leads was considered if QRS amplitude was $\leq 0.5 \mathrm{mV}$ in six limb leads. 5) Poor $R$ wave progression was diagnosed if the $S$ wave was the dominant deflection in the QRS complex, i.e. in rS waveform, from right to left precordial leads and if the ratio of R/S was < 1 in V1-V4 or beyond. 6) Presence of ischemic ST-T changes such as ST elevation accompanying T wave inversion, or ST depression with upright $\mathrm{T}$ wave in the same lead. For patients with injury or ischemic ST-T changes, short term follow-up ECGs were evaluated to determine whether these ST-T changes were resolved.

\subsection{QRSd-EF relationship}

Cardiac output was estimated by one of two methods: 1) 2-D echocardiography using modified Simpson's biplane method, or 2) post-stress, nuclear perfusion imaging the single photon emission computed tomography (gated SPECT). The QRSd was obtained from automated interpretation of each ECG tracing and QRSd-EF curve was plotted.

\subsection{Statistical analysis}

Comparisons of CAD and non-CAD groups were performed using NCSS 2007 biomedical statistical software (Kaysville, UT). A two-sample $t$ test was applied for descriptive analysis in quantitative measures showing normal distribution of data points. Nonparametric tests Mann-Whitney $U$ or Wilcoxon Rank Tests were used for difference in medians if the normality was rejected. A chi square (X2) statistic was used to compare categorical variables. For the ECG changes that were more prevalent in one group than the other, sensitivity, specificity, positive and negative predictive values were calculated for the assessment of predictive accuracy. Univariate linear regression analysis was performed when appropriate. A p value $<0.05$ was considered statistically significant.

\section{Results}

LBBB was found in 3.7\% (134/3582) of all patients screened. Among LBBB patients, $72 \%$ (96/134) had evidence of CAD. Patients with CAD were older (76 \pm 9 vs. $65 \pm 13$ years, $p$ $<0.001)$ and more commonly of male gender ( $53 \%$ vs. $43 \%$, p < 0.05$)$.

\subsection{ECG patterns}

Table 1 demonstrates that in patients with LBBB, leftward QRS axis, QS wave in V1, poor R wave progression, and broad/notched/slurred $\mathrm{R}$ wave in $\mathrm{V} 6$ are prevalent in both CAD and non-CAD groups. RS morphologies (rS, rs or Rs) in V6 was the second most common waveform (Figure 1) next to broad/notched/slurred $R$ wave in this lead, seen in $40 \%$ of $\mathrm{CAD}$ and $26 \%$ of non-CAD patients. Though it is less frequent, low QRS voltage is more common in CAD patients. Ischemic ST-T changes were infrequently seen in the CAD group. Among all ECG variables assessed, none reached statistical significance except $T$ wave inversion in $\mathrm{V} 4$, present in $13.5 \%$ in CAD and in none of the non-CAD patients ( $p<0.05)$. Clinical assessment of those with $\mathrm{T}$ wave inversion in $\mathrm{V} 4$ revealed that those patients were indeed having acute coronary events at the time the ECGs were taken. Since acute ischemic ST-T changes were infrequently seen in the CAD group, the sensitivity and negative predictive value (NPV) were low though the specificity and positive predictive value (PPV) were relatively high (Table 2). 


\begin{tabular}{r|ccc}
\hline QRS-ST-T Morphology & CAD & Non-CAD & p Value \\
\hline QRSd; ms & $148[142 ; 154]^{*}$ & $140[136 ; 150]$ & NS \\
Qeft axis deviation $\left(-30^{\circ}\right.$ to $\left.-60^{\circ}\right)$ & $54 \%$ & $42 \%$ & NS \\
QS in 1 & $62.5 \%$ & $68 \%$ & NS \\
Low QRS voltage & $11 \%$ & $5 \%$ & NS \\
Poor R progression & $86 \%$ & $87 \%$ & NS \\
Broad/notched/ slurred R wave in V6 & $60 \%$ & $74 \%$ & NS \\
T wave inversion in V2-3 & $5 \%$ & 0 & NS \\
QRS in LAFB pattern & $21 \%$ & $24 \%$ & NS \\
T wave inversion in V4 & $13.5 \%$ & 0 & $<0.05$ \\
ST elevation with inverted T wave & $5 \%$ & 0 & NS \\
ST depression with upright T wave & $16 \%$ & $8 \%$ & NS \\
\hline
\end{tabular}

*Median [95\% LCL; UCL]

Table 1. QRS-ST-T morphology in LBBB patients with and without CAD

\begin{tabular}{l|cccc}
\hline \multicolumn{1}{c|}{ ECG changes } & Sensitivity & Specificity & PPV & NPV \\
\hline T wave inversion in V2-3 & $30 \%$ & $100 \%$ & $100 \%$ & $5.2 \%$ \\
T wave inversion in V4 & $32 \%$ & $100 \%$ & $100 \%$ & $13.5 \%$ \\
ST elevation with inverted T wave & $30 \%$ & $100 \%$ & $100 \%$ & $5.2 \%$ \\
ST depression with upright T wave & $31 \%$ & $83 \%$ & $93 \%$ & $15.6 \%$ \\
\hline
\end{tabular}

PPV: positive predictive value; NPV: negative predictive value

Table 2. The predictive accuracy of CAD by presence of ischemic ST-T changes

\subsection{QRSd-EF relationship}

The median QRSd values (Table 1) in our study cohort were well above $120 \mathrm{~ms}$, indicating all patients had complete LBBB. The EF values $(0.35 \pm 0.15$ vs $0.39 \pm 0.17, \mathrm{p}=\mathrm{NS})$ were similar between $\mathrm{CAD}$ and non-CAD groups. Figures $2 \mathrm{~A}-\mathrm{B}$ demonstrate that an increased QRSd was inversely related to $\mathrm{EF}$ in both the $\mathrm{CAD}$ (Slope $=-0.36, \mathrm{R}^{2}=0.1$, correlation $=-0.319$, $\mathrm{p}<0.005$ ) and the non-CAD groups (Slope $=-0.52, \mathrm{R} 2=0.37$, correlation $=-0.61, \mathrm{p}<0.001$ ).

\section{Discussion}

CAD is one of the most common findings in patients with LBBB. Recognizing CADassociated ECG changes is of clinical importance. Among all the criteria proposed, (Havelda, Sohi et al. 1982; Fesmire 1995; Sgarbossa, Pinski et al. 1996) the Sgarbossa criteria (Sgarbossa, Pinski et al. 1996) seem very promising with good predictive values reported by the investigators. Since it is mainly targeted at ST-T changes at an acute or sub-acute phase 
of myocardial infarction, when applied to identify both acute and chronic ischemia, a low sensitivity was reported (Shlipak, Lyons et al. 1999; Gunnarsson, Eriksson et al. 2001).

Our study examined ECG changes associated with acute coronary syndromes (ACS) as well as in patients with stable CAD. Consistent with previous studies, there were no QRS-ST-T pattern differences between the patients with and without CAD.

Regardless of etiology poor $\mathrm{R}$ wave progression is highly prevalent in our study cohort (Table 1, Figure 1).

This ECG wave pattern reflects the right ventricle to septum and to left ventricle activation sequence in LBBB. Reversal of this pattern has been used in evaluating the effectiveness of CRT.(Sweeney, van Bommel et al.) Although broad/notched/slurred $\mathrm{R}$ waves are predominant in $\mathrm{V} 6$, we found that RS morphology (rS, rs or Rs) is the second most common waveform in $\mathrm{V} 6$, seen in $40 \%$ of CAD patients and $26 \%$ of non-CAD patients with LBBB. rS in V6, is part of poor R progression and can be well explained by the right to left activation sequence. (Sweeney, van Bommel et al.)

\section{QRSd vs EF}

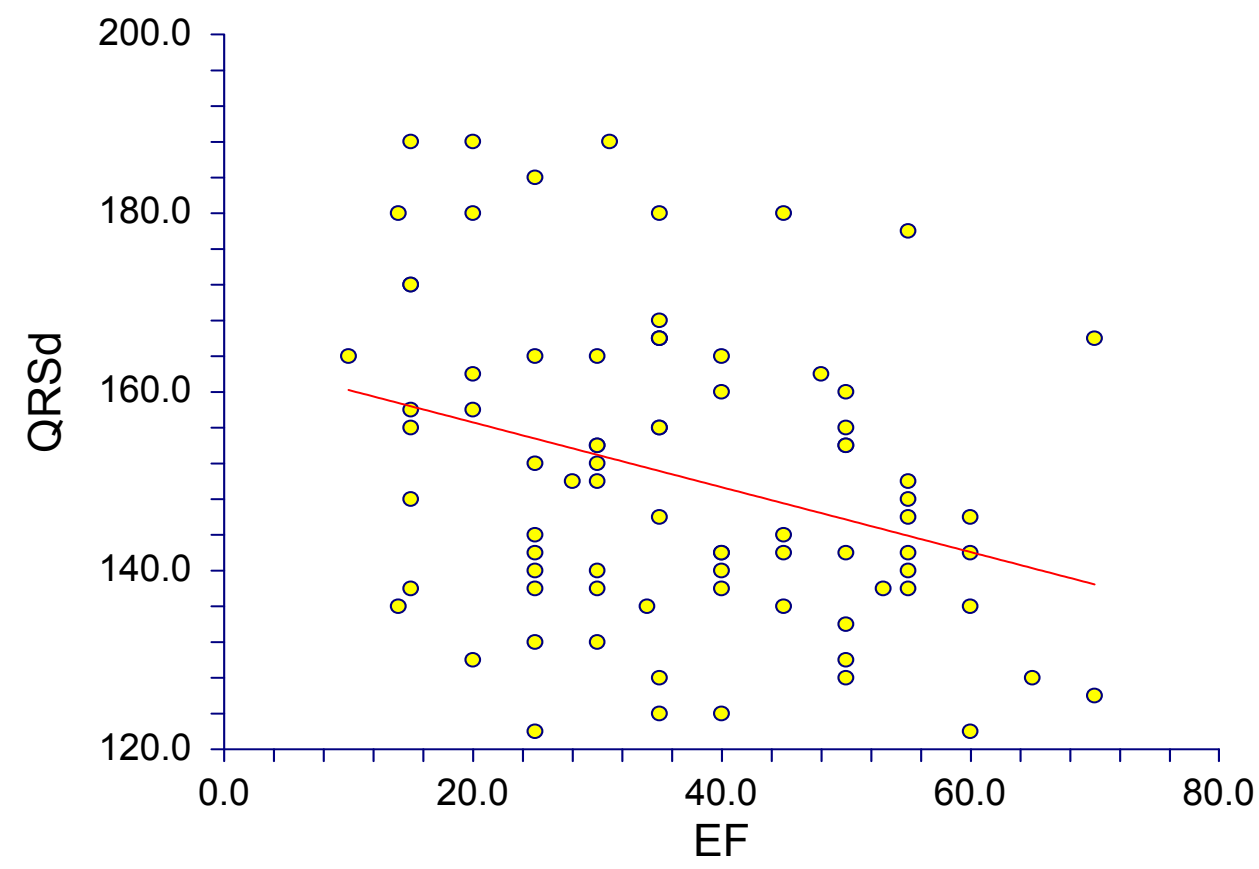

A. LBBB with CAD: QRSd $=(164)+(-0.36) \mathrm{EF}$; the correlation between QRSd and EF is -0.32 ; the estimated slope is -0.36 [ $95 \%$ CI: -0.60 lower limit; -0.12 upper limit]; the estimated intercept is 164 [95\% CI: 154 lower limit; 173 upper limit]. 


\section{QRSd vs EF}

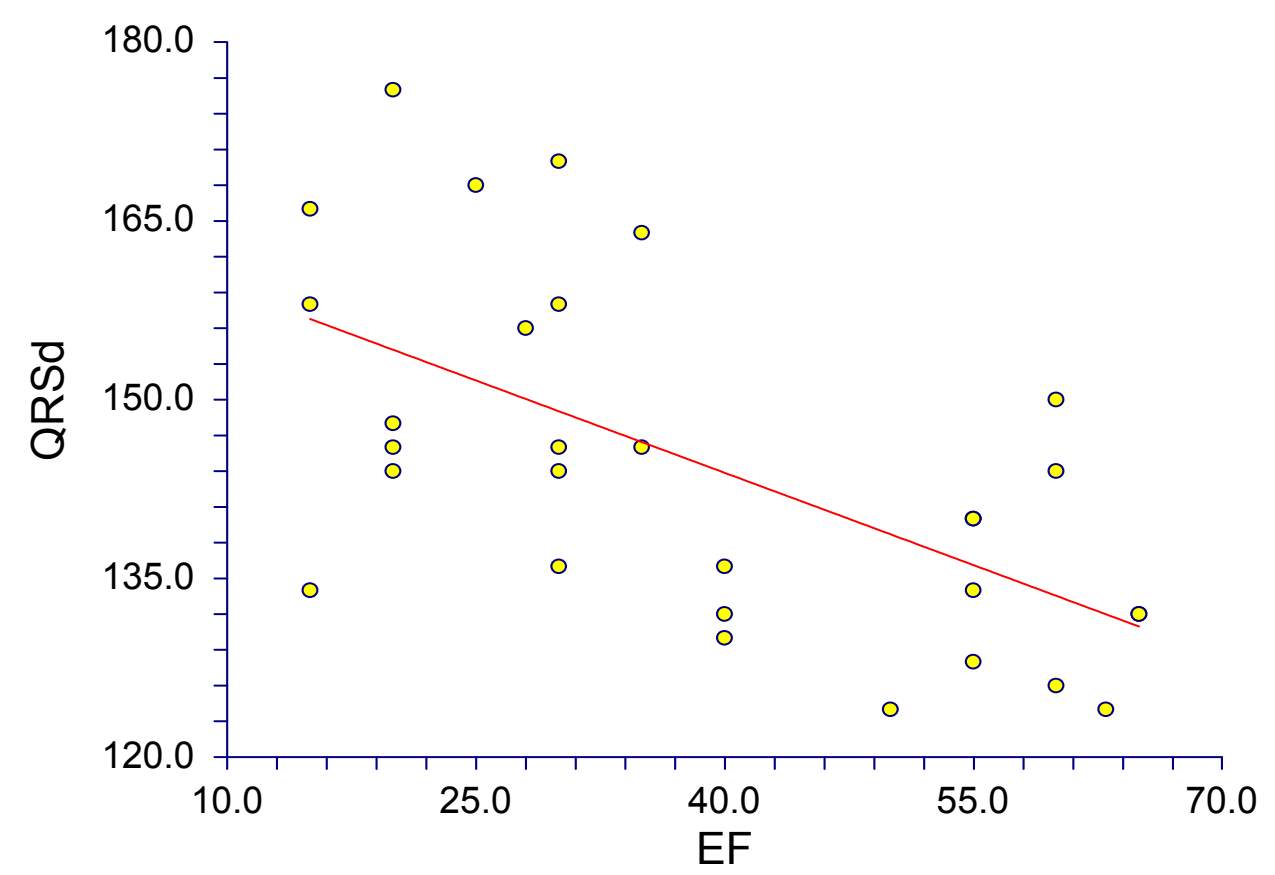

B. LBBB without CAD: QRSd $=(164)+(-0.51) \mathrm{EF}$; the correlation between QRSd and EF is -0.31 ; the estimated slope is -0.51 [ $95 \%$ CI: -0.77 lower limit; -0.36 upper limit]; the estimated intercept is 164 [95\% CI: 154 lower limit; 175 upper limit].

Fig. 2. QRSd-EF relationship in LBBB patients with and without CAD.

Depending on the blocking sites LBBB can be divided into pre-divisional, divisional and intramural subtypes.(Runge, Dorner et al. 1973; Jazayeri, Caceres et al. 1989; Childers, Lupovich et al. 2000) Pre-divisional block is at the level of left bundle trunk, divisional is at fascicular level and intramural at the intramyocardial Purkinje network. The lower level block is often associated with myocardial involvement and worse prognosis. In our study cohort, $21 \%$ of CAD and $24 \%$ non-CAD patients had LAFB ECG pattern, indicating the presence of divisional LBBB with complete blockage at LAF and partial blockage at left posterior fascicular branch level. Together they account for $42.6 \%$ of LBBB patients with leftward QRS axis.

Ischemic ST-T changes were only seen in patients with ACS. Since the majority of CAD patients do not have ACS at the time ECGs are taken, the sensitivity and negative predictive value (NPV) are low though the specificity and positive predictive value (PPV) are high (Table 2).

The second part of this study examined the correlation between QRS duration and LVEF in LBBB patients with and without CAD. LBBB patients are more predisposed to having LV 
systolic dysfunction.(Littmann and Symanski 2000; Talreja, Gruver et al. 2000) LBBB patients also have a higher mortality(Huvelle, Fay et al.) when compared to patients who also have LV systolic dysfunction without LBBB.(Abdel-Qadir, Tu et al.) This in part can be attributed to the higher than normal incidence of $\mathrm{CAD}$, hypertension, cardiomyopathy and valvular heart disease in patients with LBBB.(Abdel-Qadir, Tu et al.) These co-morbidities may be responsible for the LBBB itself. However, LBBB results in alteration of both the systolic and diastolic properties of the LV. The alterations in the diastolic filling time and septal wall motion cause both systolic and diastolic dysfunction (Grines, Bashore et al. 1989), therefore reduce the global cardiac performance. This effect, as shown in this study, is possibly exaggerated by and is positively proportional to the QRS duration. Moreover, we demonstrated that the negative correlation between QRS duration and LVEF is present in both patients with and without CAD, further proof of the negative outcome independently caused by LBBB.

\section{Conclusions}

Other than acute ischemic ST-T changes seen in patients with ACS, the overall LBBB morphology is not different in patients with and without CAD. Increased QRS duration in LBBB has a significant negative correlation with LVEF in both CAD and non-CAD patients.

\section{Acknowledgement}

Dr. Li Zhang is supported by William Wikoff Smith Charitable Trust and AHA 0735474N.

\section{References}

Abdel-Qadir, H. M., J. V. Tu, et al. "Bundle branch block patterns and long-term outcomes in heart failure." Int J Cardiol 146(2): 213-8.

Childers, R., S. Lupovich, et al. (2000). "Left bundle branch block and right axis deviation: a report of 36 cases." J Electrocardiol 33 Suppl: 93-102.

Eriksson, P., P. O. Hansson, et al. (1998). "Bundle-branch block in a general male population: the study of men born 1913." Circulation 98(22): 2494-500.

Fesmire, F. M. (1995). "ECG diagnosis of acute myocardial infarction in the presence of left bundle-branch block in patients undergoing continuous ECG monitoring." Ann Emerg Med 26(1): 69-82.

Grines, C. L., T. M. Bashore, et al. (1989). "Functional abnormalities in isolated left bundle branch block. The effect of interventricular asynchrony." Circulation 79(4): 845-53.

Gunnarsson, G., P. Eriksson, et al. (2001). "ECG criteria in diagnosis of acute myocardial infarction in the presence of left bundle branch block." Int J Cardiol 78(2): 167-74.

Havelda, C. J., G. S. Sohi, et al. (1982). "The pathologic correlates of the electrocardiogram: complete left bundle branch block." Circulation 65(3): 445-51.

Hayat, S. A., G. Dwivedi, et al. (2008). "Effects of left bundle-branch block on cardiac structure, function, perfusion, and perfusion reserve: implications for myocardial contrast echocardiography versus radionuclide perfusion imaging for the detection of coronary artery disease." Circulation 117(14): 1832-41.

Huvelle, E., R. Fay, et al. "Left bundle branch block and mortality in patients with acute heart failure syndrome: a substudy of the EFICA cohort." Eur J Heart Fail 12(2): 156-63. 
Jain, A. C. and M. C. Mehta (2003). "Etiologies of left bundle branch block and correlations with hemodynamic and angiographic findings." Am J Cardiol 91(11): 1375-8.

Jazayeri, M. R., J. Caceres, et al. (1989). "Electrophysiologic characteristics of sudden QRS axis deviation during orthodromic tachycardia. Role of functional fascicular block in localization of accessory pathway." J Clin Invest 83(3): 952-9.

Littmann, L. and J. D. Symanski (2000). "Hemodynamic implications of left bundle branch block." J Electrocardiol 33 Suppl: 115-21.

Nisse, B. S. (1929). "Congenital Heart Disease associated with Arborization Block of the Left Branch Bundle." Proc R Soc Med 22(4): 405-6.

Roshan, J., P. K. Pati, et al. (2008). "Echocardiographic evaluation of ventricular dyssynchrony in patients with left bundle branch block." Indian Heart J 60(6): 56773.

Runge, M., V. Dorner, et al. (1973). "[Predivisional left bundle-branch block with left anterior hemiblock]." Z Kardiol 62(11): 1004-12.

Sgarbossa, E. B., S. L. Pinski, et al. (1996). "Electrocardiographic diagnosis of evolving acute myocardial infarction in the presence of left bundle-branch block. GUSTO-1 (Global Utilization of Streptokinase and Tissue Plasminogen Activator for Occluded Coronary Arteries) Investigators." N Engl J Med 334(8): 481-7.

Shlipak, M. G., W. L. Lyons, et al. (1999). "Should the electrocardiogram be used to guide therapy for patients with left bundle-branch block and suspected myocardial infarction?" JAMA 281(8): 714-9.

Strauss, D. G., R. H. Selvester, et al. "Defining left bundle branch block in the era of cardiac resynchronization therapy." Am J Cardiol 107(6): 927-34.

Sweeney, M. O., R. J. van Bommel, et al. "Analysis of ventricular activation using surface electrocardiography to predict left ventricular reverse volumetric remodeling during cardiac resynchronization therapy." Circulation 121(5): 626-34.

Talreja, D., C. Gruver, et al. (2000). "Efficient utilization of echocardiography for the assessment of left ventricular systolic function." Am Heart J 139(3): 394-8. 


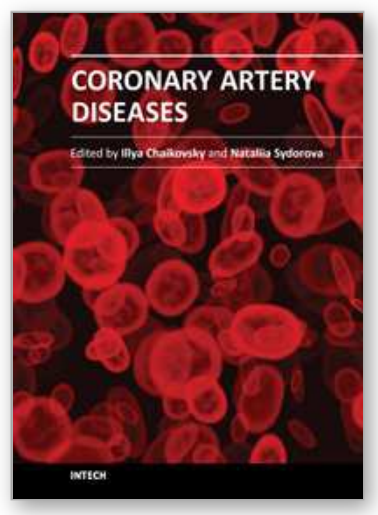

\author{
Coronary Artery Diseases \\ Edited by Dr. Illya Chaikovsky
}

ISBN 978-953-51-0238-0

Hard cover, 332 pages

Publisher InTech

Published online 07, March, 2012

Published in print edition March, 2012

This book has "wide geography" both literally and figuratively. First of all, this book brings together contributions from around the world, both from post-industrial countries and developing world. This is natural, because coronary artery disease is becoming pandemic worldwide. CAD is the single most frequent cause of death in developed countries, causes about 1 in every 5 deaths. Mortality from cardiovascular disease is predicted to reach 23.4 million in 2030. Moreover, in the developing world, cardiovascular disease tends to affect people at a younger age and thus could negatively affect the workforce and economic productivity. The morbidity, mortality, and socioeconomic importance of CAD make its diagnosis and management fundamental for all practicing physicians. On another hand, the book widely represents "geography" of CAD itself, i.e. many various aspects of its pathophysiology, epidemiology, diagnosis, treatment are touched in this book. This book does not pretend on complete and integral description of the Coronary artery disease. Rather, it contains selected issues on this complex multifactorial disease. Nevertheless, we hope that readers will find Coronary Artery Disease useful for clinical practice and further research.

\title{
How to reference
}

In order to correctly reference this scholarly work, feel free to copy and paste the following:

Marwan Badri, William Kornberg, James F. Burke, Peter R. Kowey and Li Zhang (2012). LBBB: The ECG Patterns and Cardiac Function in Patients With and Without Coronary Artery Disease, Coronary Artery Diseases, Dr. Illya Chaikovsky (Ed.), ISBN: 978-953-51-0238-0, InTech, Available from: http://www.intechopen.com/books/coronary-artery-diseases/lbbb-the-ecg-patterns-and-cardiac-function-inpatients-with-and-without-coronary-artery-disease

\section{INTECH}

open science | open minds

\section{InTech Europe}

University Campus STeP Ri

Slavka Krautzeka 83/A

51000 Rijeka, Croatia

Phone: +385 (51) 770447

Fax: +385 (51) 686166

www.intechopen.com

\section{InTech China}

Unit 405, Office Block, Hotel Equatorial Shanghai

No.65, Yan An Road (West), Shanghai, 200040, China

中国上海市延安西路65号上海国际贵都大饭店办公楼 405 单元

Phone: +86-21-62489820

Fax: $+86-21-62489821$ 
(C) 2012 The Author(s). Licensee IntechOpen. This is an open access article distributed under the terms of the Creative Commons Attribution 3.0 License, which permits unrestricted use, distribution, and reproduction in any medium, provided the original work is properly cited. 